This item was submitted to Loughborough's Research Repository by the author.

Items in Figshare are protected by copyright, with all rights reserved, unless otherwise indicated.

\title{
Adaptive soft-constraint satisfaction (SCS) algorithms for fractionally-spaced blind equalizers
}

PLEASE CITE THE PUBLISHED VERSION

PUBLISHER

(c) IEEE

VERSION

VoR (Version of Record)

LICENCE

CC BY-NC-ND 4.0

\section{REPOSITORY RECORD}

Baykal, Buyurman, Oguz Tanrikulu, and Jonathon Chambers. 2019. "Adaptive Soft-constraint Satisfaction (SCS) Algorithms for Fractionally-spaced Blind Equalizers”. figshare. https://hdl.handle.net/2134/5861. 
This item was submitted to Loughborough's Institutional Repository (https://dspace.lboro.ac.uk/) by the author and is made available under the following Creative Commons Licence conditions.

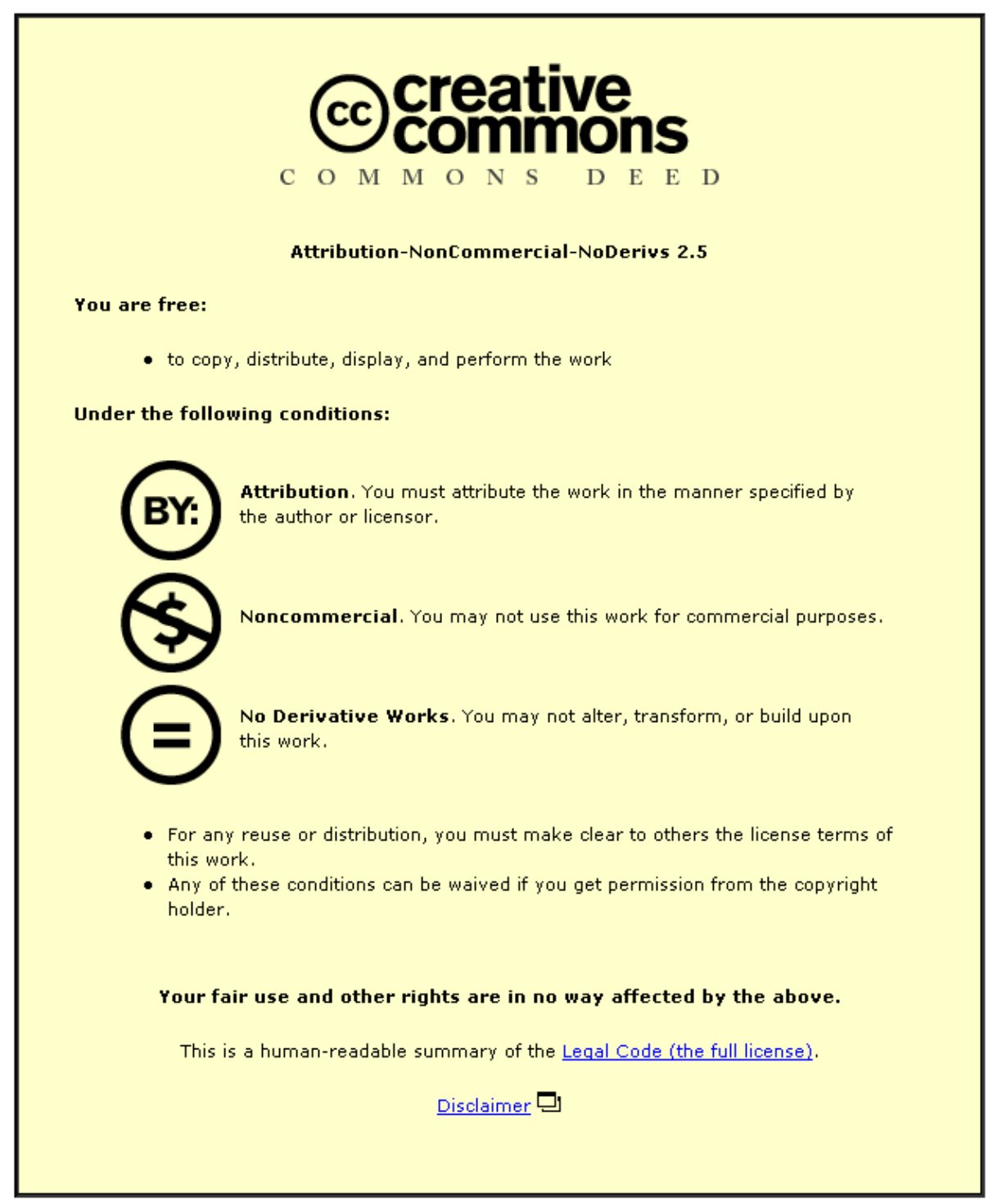

For the full text of this licence, please go to: http://creativecommons.org/licenses/by-nc-nd/2.5/ 


\title{
ADAPTIVE SOFT-CONSTRAINT SATISFACTION (SCS) ALGORITHMS FOR FRACTIONALLY-SPACED BLIND EQUALIZERS
}

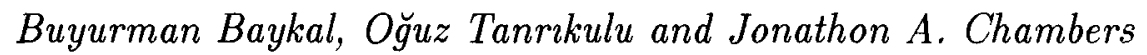 \\ Department of Electrical and Electronic Engineering, \\ Imperial College of Science, Technology and Medicine, London SW7 2BT, UK \\ E-mail: b.baykal@ic.ac.uk
}

\begin{abstract}
Constant Modulus algorithms based on a deterministic error criterion are presented. Soft constraint satisfaction methods yield a general family of blind equalization algorithms employing nonlinear functions of the equalizer output which must satisfy certain conditions. The algorithms are also extended to cover fractionally-spaced blind equalization. A normalization factor which appears as a result of the deterministic formulation of the problem helps the blind equalizer improve its performance. Also, the family supports a wide range of nonlinear functions. Extensive simulations are presented to reveal convergence characteristics which also include signals from the Signal Processing Information Base (SPIB).
\end{abstract}

\section{INTRODUCTION}

Blind equalization has been the focus of extensive research effort $[1,2,3]$ because the need to transmit a training sequence so that the equalizer can remove the effect of Inter-Symbol-Interference (ISI). Unless combatted, ISI causes decision errors in receivers. So far numerous problems, the most serious of which is the existence of undesirable local minima [4], have impeded the exploitation of blind equalizers in many commercial systems. Recently, it has been shown that [5] globally convergent blind equalizers can be built within the fractionally-spaced configuration. However, important aspects such as the numerical conditioning for various nonlinear functions of the output and stability associated with the power level of the input require further investigation.

In this paper, we develop a general family of blind equalization algorithms which is less sensitive to input power, local variations at the channel output and has flexibility in choosing the nonlinear function. Figure 1 shows a general blind equalizer. We assume that the rate of transmitted symbols, $s_{k}$, is $T$. If $p=1$, then the equalizer is single channel and termed " $T$-spaced" since the channel output is sampled at the symbol rate. If $p \geq 2$, we have a fractionally-spaced equalizer which corresponds to sampling the channel output at a rate $T / p$. In general, it is necessary to oversample the channel output at a frequency greater than the bandwidth of the transmit pulses, which is generally higher than the Nyquist rate due to pulse shaping, so that undesirable effects in $T$-sampled equalization do not exist.

\section{DETERMINISTIC OPTIMIZATION CRITERION}

Blind equalization algorithms can be derived from a deterministic optimization criterion. Consider the following optimization problem, also known as the principle of minimum disturbance [6]: Determine the tapweight vector of dimension $N$ at time $k, W_{k}$, given the tap-input vectors $X_{k}, X_{k-1}, \ldots$, where $X_{k}^{H}=$ $\left[\begin{array}{lll}x_{k} & \ldots & x_{k-N+1}\end{array}\right]$, and desired responses $d_{k}, d_{k-1}, \ldots$, so as to minimize the squared Euclidean norm of the change in the tap-weight vector $W_{k}$,

$$
\delta W_{k}=W_{k}-W_{k-1}
$$

subject to the constraints

$$
\begin{aligned}
X_{k}^{H} W_{k} & =d_{k} \\
X_{k-1}^{H} W_{k} & =d_{k-1} \\
& \vdots \\
X_{k-m+1}^{H} W_{k} & =d_{k-m+1},
\end{aligned}
$$

where $m<N$ and $(.)^{H}$ denotes Hermitian transpose. If the training sequence is known to the receiver, the solution of the optimization problem leads to the Underdetermined Recursive Least-Squares (URLS) algorithm [7], also known as the Affine Projection algorithm. In blind equalization the training sequence is not known to the receiver, and hence the desired response must be obtained from pertinent measurements in the receiver. In this paper, we propose the following choice 
for $d_{k} \ldots d_{k-m+1}$ :

$$
\begin{aligned}
d_{k} & =\psi\left(R, y_{[k-1], k}\right) \\
& \vdots \\
d_{k-m+1} & =\psi\left(R, y_{[k-1], k-m+1}\right)
\end{aligned}
$$

where $y_{[i], j}=W_{i}^{H} X_{j}, \psi($.$) is a nonlinear function$ which satisfies certain conditions and $R$ is a constant chosen appropriately for the particular modulation scheme employed in the transmission. In the sequel we use $y_{k}$ to denote $y_{[k-1], k}$. The solution of the above optimization problem via Lagrange multipliers yields

$$
W_{k}=W_{k-1}+\mathbf{X}_{m, k}\left(\mathbf{X}_{m, k}^{H} \mathbf{X}_{m, k}\right)^{-1} \mathcal{E}_{k},
$$

where the $m \times 1$ error vector $\mathcal{E}_{k}$ and the $N \times m$ matrix (vector aggregate of $X_{k}$ ) $\mathbf{X}_{m, k}$ are

$$
\begin{aligned}
& \mathbf{X}_{m, k} \triangleq\left[\begin{array}{llll}
X_{k} & X_{k-1} & \cdots & X_{k-m+1}
\end{array}\right], \\
& \mathcal{E}_{k} \triangleq \mathcal{D}_{k}-\mathbf{X}_{m, k}^{H} W_{k-1}, \\
& \mathcal{D}_{k} \triangleq\left[\psi\left(R, y_{[k-1], k}\right) \cdots \psi\left(R, y_{[k-1], k-m+1}\right)\right]^{H} \text {. }
\end{aligned}
$$

A stepsize is also introduced to maintain the stability of the algorithm. Hence, we have the update

$$
W_{k}=W_{k-1}+\mu \mathbf{X}_{m, k}\left(\mathbf{X}_{m, k}^{H} \mathbf{X}_{m, k}\right)^{-1} \mathcal{E}_{k} .
$$

A similar formulation has also appeared in [3] where only the signum function is considered in $\mathcal{D}$ and the resulting algorithm is interpreted as a projection onto a circle. The family of functions which satisfy the requirements for convergence constitutes the soft-constraint satisfaction algorithms because at each iteration the constraints in (2) are dynamically changed. Some special cases may be identified: For $m=1, R=E\left\{\left|s_{k}\right|^{2 p}\right\} / E\left\{\left|s_{k}\right|^{p}\right\}$ and $\psi\left(R, y_{k}\right)=$ $y_{k}\left(R\left|y_{k}\right|^{(p-2)}-\left|y_{k}\right|^{(2 p-2)}+1\right)$ we can identify the normalized version of the Godard algorithm [1]. For $m=1, R=E\left\{\left|s_{k}\right|^{2}\right\} / E\left\{\left|s_{k}\right|\right\}$ and $\psi\left(R, y_{k}\right)=$ $R \operatorname{sgn}\left(y_{k}\right)$, we have the normalized version of the Sato algorithm [2]. New nonlinear functions can also be introduced. For example, when $m=1, R=$ $E\left\{\left|s_{k}\right|^{3}\right\} / E\left\{\left|s_{k}\right|^{2}\right\}$ and $\psi\left(R, y_{k}\right)=\left(2-\left|y_{k}\right| / R\right) y_{k}$, the SCS-1 algorithm in [8] results. Another choice could be $R^{1 / 2}=E\left\{\left|s_{k}\right|^{2}\right\} / E\left\{\left|s_{k}\right|^{3 / 2}\right\}$ and $\psi\left(R, y_{k}\right)=$ $R^{1 / 2} \operatorname{sgn}\left(y_{k}\right)\left|y_{k}\right|^{1 / 2}$, which will be termed as the Squareroot SCS (SqSCS) algorithm.

The methodology in this section is reminiscent of Bussgang techniques for blind equalization, for which the memoryless nonlinear function $\psi($.$) is thought of$ estimating the conditional mean $E\left\{s_{k} \mid y_{k}\right\}$ [9]. If a deterministic formulation of the problem is adopted, the factor $\left(\mathbf{X}_{m, k}^{H} \mathbf{X}_{m, k}\right)^{-1}$ can significantly affect the performance as shown in [8]. The simulations presented in this paper also illustrate this fact.

\section{FRACTIONALLY-SPACED EQUALIZERS}

The concept of soft constrained satisfaction can be extended to fractionally-spaced equalizers. The SingleInput-Multiple-Output (SIMO) system of Figure 1 represents a fractionally sampled equalizer. The optimization problem of the previous section can be extended to cover the multichannel setup. In this case, let $W_{k}^{H}=\left[W_{k}^{(1) H} \cdots W_{k}^{(p) H}\right], X_{k}^{H}=\left[X_{k}^{(1) H} \cdots X_{k}^{(p) H}\right]$ and $y_{k}=X_{k}^{H} W_{k-1}$. The nonlinear functions can be used without any alteration.

\section{SIMULATIONS}

The proposed algorithms have been tested on the artificially created data and real data sets which are being placed in the Signal Processing Information Base. In particular, the simulations with the Godard $(p=2)$, normalized Godard ( $p=2)$, normalized Sato, SCS1 and SqSCS algorithms with $m=1$ have been presented. The step-sizes of all algorithms have been chosen to give the fastest convergence in each case.

\subsection{Artifically Created Data Sets}

We have used a 2-channel SIMO structure to simulate a fractionally-spaced blind equalizer which has an oversampling factor of 2. BPSK modulation technique is assumed.

Experiment I: The mixed-phase subchannels in the upper and lower branches of the communication channel are respectively chosen to be $0.242,-0.204,-0.159$, $0.142,0.157$ and $0.216,0.508,0.848,0.530,0.311$. The equalizer has 4 taps in each subchannel. All algorithms start from the same arbitrary initial condition. The signal-to-noise-ratio in each subchannel is set to $10 \mathrm{~dB}$ and the results of 20 independent trials are averaged to obtain the Open-Eye Measure (OEM) which is defined as

$$
\operatorname{OEM}(k) \triangleq \frac{\left\|T_{k}\right\|_{1}-\left\|T_{k}\right\|_{\infty}}{\left\|T_{k}\right\|_{\infty}}
$$

where $T_{k}$ represents the combined channel and equalizer. If $\operatorname{OEM}(k)<0 \mathrm{~dB}$ then the eye is open and ISI has no effect in the decision process. If $\operatorname{OEM}(k)>0 \mathrm{~dB}$, the eye is closed and hence the ISI left after equalization will cause decision errors. The evolution of the OEMs for the proposed algorithms is shown in Figure 2 .

Experiment II: In this experiment a common zero is assumed between the channels. The upper and lower 
subchannels are assumed to be $0.197,0.586,0.960$, $0.705,0.217$ and $0.179,0.422,0.706,0.440,0.2588$ respectively. There are common zeros at $1.5 e^{\mp j 0.7 \pi}$. The equalizer has 4 taps in each subchannel. Center-tap initialization is used in each subchannel. The results of 20 independent trials are averaged to obtain the OEM curves presented in Figure 3.

When common zeros exist between channels, the problem is equivalent to $T$-spaced equalization of the common transfer function [10]. Hence, the Godard algorithm may fail to converge to the global optimum which is the possible cause of slow convergence of this case in the simulations.

The experiments show that SCS algorithms perform better than the unnormalized algorithms. Also a discontinuous nonlinearity as in the Sato or Normalized Sato algorithm is not desirable. It can also be concluded that the performance is unlikely to depend on the choice of the nonlinear function. Therefore, the one with better numerical properties could be chosen.

\subsection{SPIB Signals}

In this part, the algorithms are tested with the real data sets obtained from the SPIB database defined in the appendix of [11]. A V.29 constellation modem sequence is chosen. The channel output is sampled twice faster than the symbol period. The power spectral density and constellation of the channel output are shown in Figure 4 . The equalizer has 8 taps in each subchannel. The constellations at the equalizer output in the steady-state for the Godard, Normalized Godard, SCS-1 and SqSCS algorithms can be seen in Figure 5. Although some carrier offset remains in the data set, all algorithms are able to open the channel eye.

\section{CONCLUSIONS}

A family of blind equalization algorithms is proposed for $T$-spaced and fractionally spaced equalizers. Better performance in realistic situations, flexibility in choosing the nonlinear function and less sensitivity to the input power level are the essential features of the new family.

\section{REFERENCES}

[1] N. Godard, "Self-recovering equalisation and carrier tracking in two-dimensional data communications systems," IEEE Trans. Commun., vol. COM-28, no. 10, pp. $1867-1875,1980$.

[2] Y. Sato, "A method of self-recovering equalisation for multilevel amplitude modulation systems," IEEE Trans. Commun., vol. COM-23, pp. 679-682, June 1975.
[3] C. Papadias, Methodés d'égalisation et d'identification aveugle pour les canaux linéaires. $\mathrm{PhD}$ thesis, Ecole Nationale Supérieure des Télécommunications, May 1995.

[4] Z. Ding, C. Johnson Jr., and R. Kennedy, "On the (non)existance of undesirable equilibria of Godard blind equalisers," IEEE Trans. Sig. Proc., vol. SP-40, pp. 2425-2432, Oct. 1992.

[5] Y. Li and Z. Ding, "Global convergence of fractionally spaced Godard (CMA) adaptive equalizers," IEEE Transactions on Signal Processing, vol, 44, no. 4, pp. 818-826, 1996.

[6] B. Widrow and M. Lehr, "30 years of adaptive neural networks: Perceptron, madaline and backpropagation," Proceedings of the IEEE, Special Issue on Neural Networks I, vol. 78, 1990.

[7] B. Baykal, Underdetermined Recursive-Least Squares Adaptive Filtering. $\mathrm{PhD}$ thesis, Imperial College, London, July 1995.

[8] O. Tanrikulu, A. G. Constantinides, and J. A. Chambers, "A normalised constant modulus blind equalisation algorithm via constrained optimisation," in Int. Conf. Telecomms., Istanbul, Turkey, Apr. 1996.

[9] S. Bellini, "Bussgang techniques for blind deconvolution and equalisation," in Blind Deconvolution (S. Haykin, ed.), ch. 2, pp. 8-59, Prentice-Hall, 1994.

[10] I. Fijalkow, J. Treichler, and C. Johnson Jr., "Fractionally spaced blind equalization: Loss of channel disparity," in Proceedings of the ICASSP-95, pp. 1988-1991, 1995.

[11] J. Treichler, I. Fijalkow, and C. Johnson Jr, "Fractionally spaced equalizers: How long should they really be?," IEEE Signal Processing Magazine, pp. 6581, May 1996.

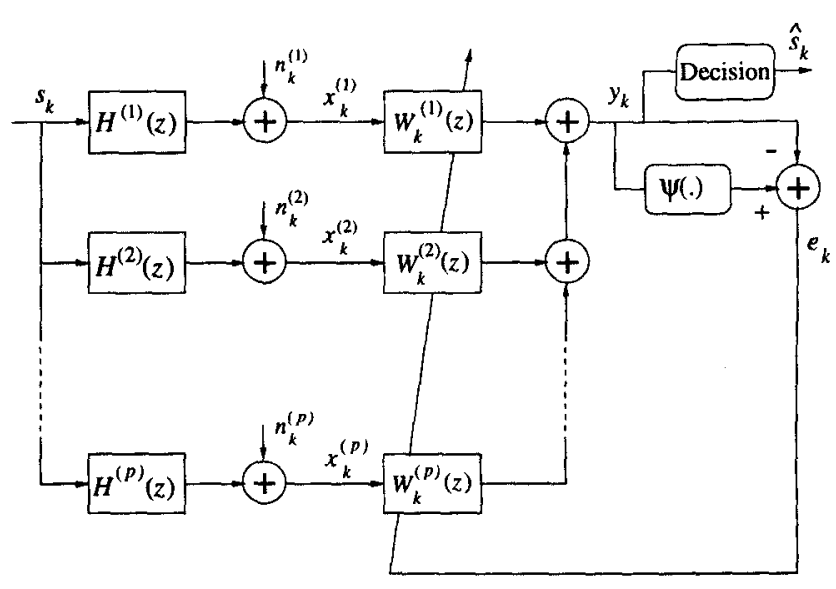

Figure 1: SIMO structure for fractionally-spaced equalization. 

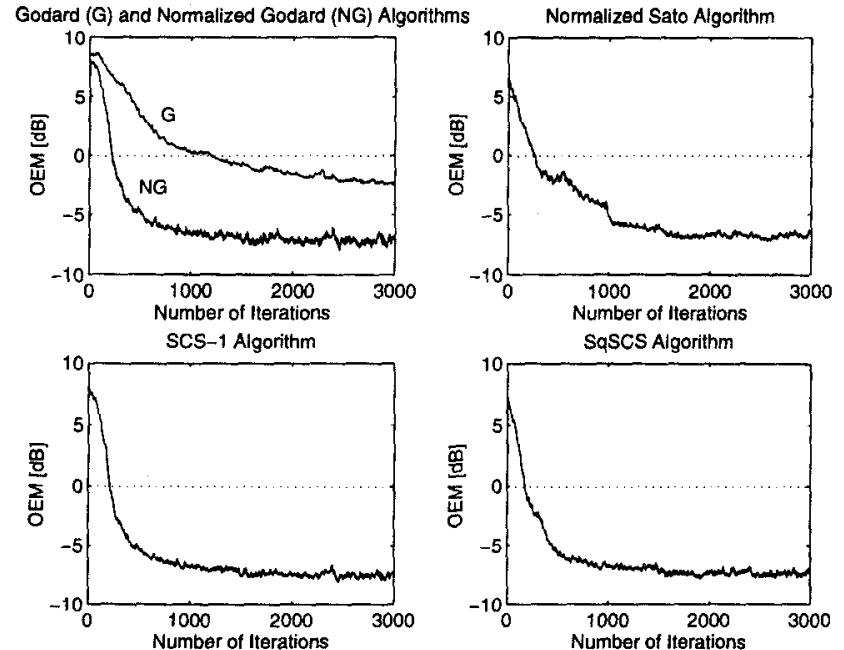

Figure 2: OEMs of all algorithms for Experiment I.

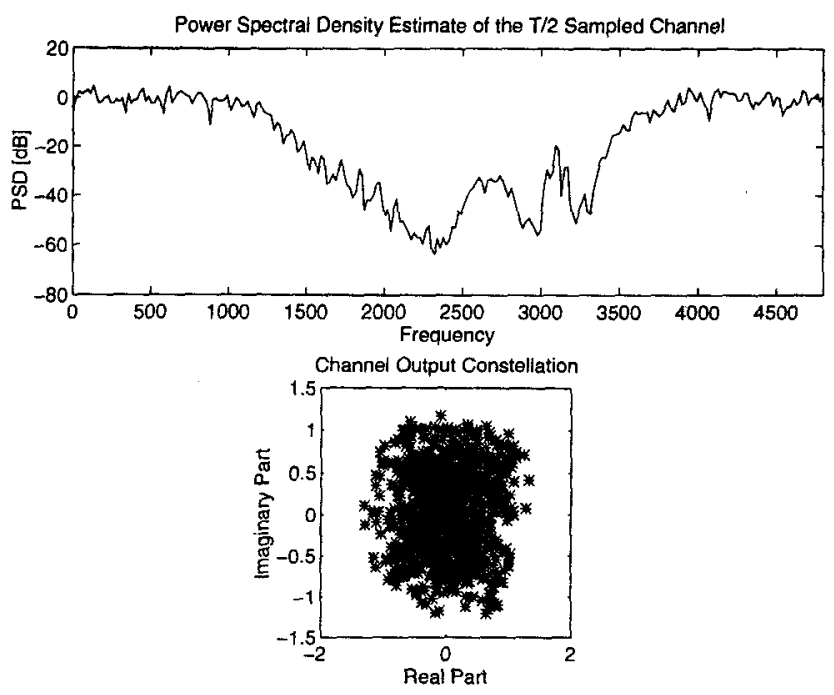

Figure 4: Characteristics of the SPIB modem channel.
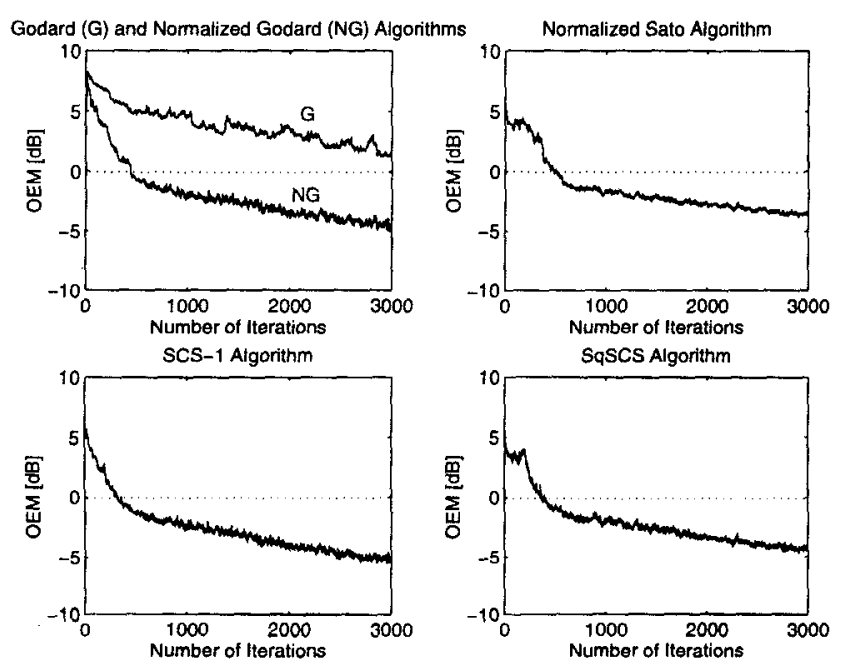

Figure 3: OEMs of all algorithms for Experiment II.
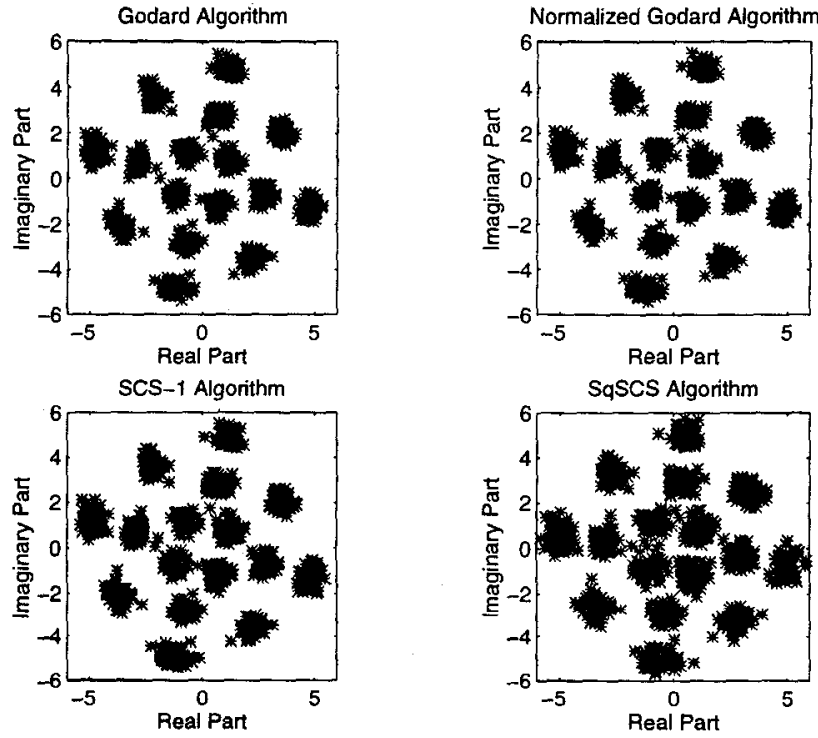

Figure 5: Output Constellations for the Godard, Normalized Godard, SCS-1 and SqSCS algorithms. 